\title{
On a conjecture of De Giorgi related to homogenization
}

\author{
Aram L. Karakhanyan ${ }^{1}$ • Henrik Shahgholian² ${ }^{2}$
}

Received: 26 August 2015 / Accepted: 17 April 2017 / Published online: 5 May 2017

(C) The Author(s) 2017. This article is an open access publication

Abstract For a periodic vector field $F$, let $X^{\varepsilon}$ solve the dynamical system

$$
\frac{\mathrm{dX}^{\varepsilon}}{\mathrm{d} t}=F\left(\frac{X^{\varepsilon}}{\varepsilon}\right) .
$$

In (Set Valued Anal 2(1-2):175-182, 1994) Ennio De Giorgi enquiers whether from the existence of the limit $X^{0}(t):=\lim _{\varepsilon \rightarrow 0} X^{\varepsilon}(t)$ one can conclude that $\frac{\mathrm{d} X^{0}}{\mathrm{~d} t}=$ constant. Our main result settles this conjecture under fairly general assumptions on $F$, which in some cases may also depend on $t$-variable. Once the above problem is solved, one can apply the result to the corresponding transport equation, in a standard way. This is also touched upon in the text to follow.

Keywords Dynamical system · ODE · Transport · Homogenization · Convergence rate Mathematics Subject Classification 34C29 $37 \mathrm{~A} 10 \cdot 65 \mathrm{~L} 70 \cdot 74 \mathrm{Q} 10$

H. Shahgholian was supported by Swedish Research Council. A. Karakhanyan was partly supported by EPSRC Grant. We thank Michael Benedicks for his insightful comments on the dynamical system issues of the current note, and Björn Engquist for bringing to our attention the paper [8].

$凶$ Henrik Shahgholian henriksh@kth.se

Aram L. Karakhanyan aram.karakhanyan@ed.ac.uk

1 School of Mathematics, The University of Edinburgh, Mayfield Road, King's Buildings, Edinburgh EH9 3JZ, UK

2 Department of Mathematics, KTH, Lindstedtsvägen 25, 10044 Stockholm, Sweden 


\section{Introduction}

\subsection{Problem setting}

For each $i=1, \ldots, d$ let $F_{i}:[0, \infty) \times \mathbb{R}^{d} \rightarrow \mathbb{R}$ be a smooth 1-periodic function for both variables. Let us consider the first-order system of differential equations with oscillating structure

$$
\frac{\mathrm{d} x_{i}}{\mathrm{~d} t}=F_{i}\left(\frac{t}{\varepsilon}, \frac{x_{1}}{\varepsilon}, \ldots, \frac{x_{d}}{\varepsilon}\right) \quad i=1, \ldots, d,
$$

where $\varepsilon>0$ is a small parameter. Our primary motivation for studying (1) comes from a conjecture posed by Ennio De Giorgi in [6] (Conjecture 1.1 page 175) concerning the homogenization of the transport equation

$$
\begin{aligned}
& \partial_{t} u^{\varepsilon}(t, x)+\mathrm{F}(t / \varepsilon, x / \varepsilon) \cdot \nabla_{x} u^{\varepsilon}(t, x)=0, \quad t \in(0, \infty), x \in \mathbb{R}^{d}, \\
& u^{\varepsilon}(t=0, x)=u_{0}(x), \quad x \in \mathbb{R}^{d},
\end{aligned}
$$

with vector field $\mathrm{F}=\left(F_{1}, F_{2}, \ldots, F_{d}\right)$ Lipschitz continuous and periodic in both variables $(t, x)$. The Lipschitz continuous initial condition $u_{0}(x)$ is specified at the initial time $t=0$.

He also conjectured that if (2) is homogenizable then the following property must be true (see [6, page 177]): Let $X^{\varepsilon}(t)$ be the solution of the following initial value problem

$$
\frac{\mathrm{d} X^{\varepsilon}}{\mathrm{d} t}=F\left(\frac{t}{\varepsilon}, \frac{X^{\varepsilon}}{\varepsilon}\right), \quad X^{\varepsilon}(0)=p
$$

for some given initial condition $p \in \mathbb{R}^{d}$. Then the limit exists

$$
X^{0}(t):=\lim _{\varepsilon \rightarrow 0} X^{\varepsilon}(t)
$$

for any $t, p$. Moreover, there is a vector $B \in \mathbb{R}^{d}$ such that

$$
\frac{\mathrm{d} X^{0}}{\mathrm{~d} t}=B
$$

We remark that Peirone [13] showed that if $F$ does not depend on $t$, then the asymptotic linearity of $X^{\varepsilon}(t)$ as $t \rightarrow \infty$ implies that (2) is homogenizable; see Remark 4.

\subsection{Related work}

In view of Peirone's result [13], the homogenization of (3) is closely related to the homogenization of the first-order transport equations $\partial_{t} u+F \cdot \nabla u=0$ describing miscible flow in porous media [18]. One of the central questions concerning (2) is the strong convergence which is not true in general as the example of Eq. (2) with $F\left(t, x_{1}, x_{2}\right)=\left(0, \sin x_{1}\right), d=2$ shows; see [6] page 176. It is known that if $\operatorname{div} F=0^{1}$ then the effective equation has arithmetic averages $\left(\int_{\mathbb{T}^{2}} F_{1}(x) \mathrm{d} x, \int_{\mathbb{T}^{2}} F_{2}(x) \mathrm{d} x\right)$ as the forcing velocity, whereas the shear field $F(x)=a \varphi(x), a=(1, \gamma) \in \mathbb{R}^{2}$ yields harmonic averages, i.e., in the homogenized equation the forcing velocity is $a \int_{\mathbb{T}^{2}} \frac{\mathrm{d} x}{\varphi(x)}$, see [18]. The interested reader can find more on this problem in the works $[5,9,18]$ and the references therein.

The homogenization of more general transport equations

$$
\partial_{t} u^{\varepsilon}+\operatorname{div}\left[a_{\varepsilon} f\left(u^{\varepsilon}\right)\right]=0, \quad u^{\varepsilon}(0, x)=U_{0}(x, x / \varepsilon)
$$

\footnotetext{
1 This refers to the case of unit density $\rho=1$ for the invariant measure; see Sect. 1.4 for more details.
} 
under the assumption $a_{\varepsilon}=a(x, x / \varepsilon)$ and $\operatorname{div}_{x} a(x, y)=\operatorname{div}_{y} a(x, y)=0$, is studied in [20]. The case when $a_{\varepsilon}=a(x / \varepsilon)$ is studied in [8]. It is also shown that solutions of (6) converge in $L^{2}$ and the limit equation is either a constant coefficient linear transport equation (ergodic case) or an infinite dimensional dynamical system, see $[8,20]$.

In [17] Tartar studied some transport equations with memory effects. He addressed the question of importance of considering the limit function rather than the equation it satisfies. The question he raised was whether the limit retains, in some sense, the structure of linear transport equations (e.g., when it is traveling wave solution).

Some of these questions were addressed by Tassa in [18]. In particular, he showed that for shear flow $(d=2)$ the limit is a traveling wave (Theorems 4.2 and 4.5 in [18]). He also derived convergence rate which depends on the smoothness of the forcing vector field as well as on whether the rotation number (which we denoted $\gamma$ in the formula $a=(1, \gamma)$ above) is rational or irrational. In fact for rational rotation number (Theorem 4.5 in [18]) the limit is determined by some function $a_{\eta}$; see (3.13) in [18], and the limit function is a traveling wave if $a_{\eta}=$ const for all $\eta \in[0,1]$.

It seems plausible that the techniques here can (partially) be applied to more general context involving random structure, i.e., stochastic differential equations. Similar type of problems has been studied in recent works of Bardi-Cesaroni-Scotti [2]. The problem here can be reduced to the well-known classical perturbation problem through variable substitution $Y^{\varepsilon}=X^{\varepsilon} / \varepsilon$. To illustrate this at a heuristic level, we assume (for clarity) $F$ to be independent of $t$. We thus have

$$
\frac{\mathrm{d} X^{\varepsilon}}{\mathrm{d} t}=F\left(Y^{\varepsilon}\right), \quad X^{\varepsilon}(0)=p \quad \Rightarrow \quad \frac{\mathrm{d} Y^{\varepsilon}}{\mathrm{d} t}=\frac{1}{\varepsilon} F\left(Y^{\varepsilon}\right), \quad Y^{\varepsilon}(0)=p / \varepsilon .
$$

Introducing $Z^{\varepsilon}(s)=Y^{\varepsilon}(\varepsilon s), s>0$, we infer $\frac{\mathrm{d} Z^{\varepsilon}}{\mathrm{d} s}=\varepsilon\left(Y^{\varepsilon}\right)^{\prime}(\varepsilon s)=F\left(Z^{\varepsilon}\right)$ and thus by Theorem 3.1 [13] we get that for a fixed $\varepsilon>0$ the limit

$$
\lim _{s \rightarrow \infty} \frac{Z^{\varepsilon}(s)}{s}=\lim _{s \rightarrow \infty} \frac{X^{\varepsilon}(\varepsilon s)}{\varepsilon s}:=B
$$

exists and is independent of $\varepsilon$ for a suitable class of $F$. If we knew that this limit is also uniform in $\varepsilon$, then for $\tau=\varepsilon s$ we could conclude that $\lim _{\varepsilon \rightarrow 0} \frac{X^{\varepsilon}(\tau)}{\tau}$ exists for each fixed $\tau$ and is independent of $\varepsilon$ or, equivalently, $X^{\varepsilon}(\tau)=o_{\varepsilon}(\tau)+B \tau$. Certainly this captures the case when $p=0$. Nevertheless, it is possible that our Theorem $2 \mathrm{~b}$ has some overlapping with above mentioned Theorem 3.1 [13].

A further direction, that our approach might be possible to extend to, is that of multiscale problems. More exactly, one may consider $\mathrm{F}$ that has both slow and fast variable $\mathrm{F}(x, x / \varepsilon)$. A particular case of this was studied by Menon [12], with $\mathrm{F}(x, x / \varepsilon)=\operatorname{div}(\mathrm{K}(x)+\varepsilon \mathrm{A}(x / \varepsilon))$.

\subsection{Problem setup}

We shall switch between cases of $t$-dependent as well as $t$-independent $F$, and this will be clear from the context. Hence we shall use both notation $F(t, x)$, as well as $F(x)$.

Next, going back to our $t$-independent $F$, one can establish a number of remarkable properties, for the non-oscillating system (i.e., when $\varepsilon=1$ )

$$
\frac{\mathrm{d} x_{i}}{\mathrm{~d} t}=F_{i}\left(x_{1}, \ldots, x_{d}\right) .
$$

Suppose that (7) has invariant measure $\mathrm{d} \mu_{x}=\rho(x) \mathrm{d} x$ with density $\rho>0$, i.e., the vector field $\rho F$ is divergence free; see Sect. 1.4 for details. For the two-dimensional problem, $(d=2)$, 
Kolmogorov proved that if $F(x)=\left(F_{1}\left(x_{1}, x_{2}\right), F_{2}\left(x_{1}, x_{2}\right)\right) \neq 0$, is $\mathbb{Z}^{2}$ periodic and both $\rho$ and $F$ are real analytic in $\left(x_{1}, x_{2}\right)$ variables, then there is an analytic transformation of coordinates $y=f(x)$ such that (7) transforms into shear flow system

$$
\frac{\mathrm{d} y_{i}}{\mathrm{~d} t}=\frac{a_{i}}{G\left(y_{1}, y_{2}\right)}, \quad i=1,2,
$$

with constants $a_{1}=1, a_{2}=\gamma \in \mathbb{R}$ and $G$ being a $\mathbb{Z}^{2}$ periodic scalar function. Here $\gamma$ is called the rotation number of (7) (also called rotation index), and the system (7) is ergodic if $\gamma$ is diophantine; see [16]. For the latter case, the shear flow (8) can be further transformed to a constant speed system $\frac{\mathrm{d} w^{i}}{\mathrm{~d} t}=c_{i}, i=1,2$ where $c_{i}$ are constants.

In fact, one can take

$$
G\left(x_{1}, x_{2}\right)=\rho\left(x_{1}, x_{2}\right), \text { for a.e. }\left(x_{1}, x_{2}\right) \in \mathbb{R}^{2}
$$

to be the density of invariant measure of (8) such that we have $\operatorname{div} \frac{\rho}{G}=0$. In other words, now $\frac{1}{G}$ is the density of the invariant measure of the new shear flow system of differential Eq. (8), obtained from (7) via a coordinate transformation introduced by Kolmogorov [10].

The main goal of this article is to analyze the behavior of the solution $X^{\varepsilon}(t)$ to Eq. (3) as $\varepsilon \rightarrow 0$ under some conditions imposed on the vector field $F=\left(F_{1}, \ldots, F_{d}\right)$ which we list below:

(F.1) $F: \mathbb{R}^{d} \rightarrow \mathbb{R}^{d}$ is continuous, $\mathbb{Z}^{d}$-periodic, and there is a constant $L>0$ such that

$$
\left|F\left(u_{1}\right)-F\left(u_{2}\right)\right| \leqslant L\left|u_{1}-u_{2}\right|, \quad \forall u_{1}, u_{2} \in \mathbb{R}^{d} .
$$

We write $F=\left(F_{1}, \ldots, F_{d}\right)$ where $F_{i}, 1 \leqslant i \leqslant d$ are the components of the vector field $F$.

(F.2) There is a constant $\lambda>0$ such that

$$
\lambda \leqslant F_{i}(u) \leqslant \frac{1}{\lambda}, \quad 1 \leqslant i \leqslant d
$$

for every $u \in \mathbb{R}^{d}$.

(F.3) There is a bounded $\mathbb{Z}^{d}$ periodic function $\rho>0$ such that $\operatorname{div}(\rho F)=0$ in $\mathbb{R}^{d}$. Here $\rho$ is called the density of invariant measure.

Equation $\operatorname{div}(\rho F)=0$ is understood in the weak sense, i.e., $\int \rho F \cdot \nabla \psi=0$ for every $\psi \in C_{0}^{\infty}\left(\mathbb{R}^{d}\right)$.

The conditions (F.1)-(F.3) will be mainly used in the statement of Theorem 2.

\subsection{Invariant measure}

(General discussion) Condition (F.3) needs some explanation. Suppose that $F(x)=$ $\frac{a}{G(x)}, x \in \mathbb{R}^{d}$ for some constant vector $a$ and suitable scalar function $G$ such that $F$ is smooth. It is clear that for this case $\rho=G$. However for general flows the existence of $\rho$ is not easily obtained. In the proof of Theorem $2 \mathrm{~b}$, we require that the invariant measure exists and is bounded in order to construct a change of variables which reduces general flows to shear one. In this regard, we mention the following existence result from [7]: Suppose $F: \mathbb{R}^{d} \rightarrow \mathbb{R}^{d}, F \in C^{1}$ and for simplicity $t$-independent. Let $\rho$ be sought as the solution of Liouville's equation $\operatorname{div}(\rho F)=0$. Let $\tau=x_{d}, x^{\prime}=\left(x_{1}, x_{2}, \ldots, x_{d-1}, 0\right)$ and assume that $F_{d}>0$ then Liouville's equation can be rewritten as follows

$$
\partial_{\tau} \log \rho+\nabla_{x^{\prime}} \log \rho \cdot \frac{F^{\prime}}{F_{d}}=-\frac{\operatorname{div} F}{F_{d}},
$$


where $F^{\prime}=\left(F_{1}, \ldots, F_{d-1}, 0\right)$. We can specify initial condition at time $\tau=0$, i.e., $x_{d}=0$, and then by [7] (Proposition II.1 and Remark afterward) there is a $L^{\infty}$-solution of this Cauchy problem in $\mathbb{R}^{d} \times[0, \infty)$, provided that both $F$ and the initial data are Lipschitz.

If $d=2$, then it is well known that divergence-free vector field is 90 degree rotation of the gradient of a potential function $u$, i.e., $\rho F=\left(u_{x_{2}},-u_{x_{1}}\right)$. From here we have that $\rho F_{1}=u_{x_{2}}, \rho F_{2}=-u_{x_{1}}$. For $F$ satisfying (F.2), we can eliminate $\rho$ to obtain

$$
u_{x_{1}}=-u_{x_{2}} \frac{F_{2}}{F_{1}} \text { in } \mathbb{T}^{2} .
$$

The existence and regularity of periodic solution $u=u\left(x_{1}, x_{2}\right)$ follow from standard existence theory for the first-order linear equations via the method of characteristics. In particular, if $F \in C^{k}$, then $\nabla u \in C^{k}$. The density of the invariant measure can be recovered as follows $\rho=\frac{u_{x_{1}} F_{2}-u_{x_{2}} F_{1}}{|F|^{2}}$.

\subsection{The approach and methodology}

De Giorgi's conjecture has (more or less) been ignored completely. Indeed, the fact that convergence of the underlying dynamical system would give the convergence of the transport problem, have been unnoticed in the literature. Our result (read observation) should be seen in the light of homogenization of the dynamical system, rather than the transport problem, even though this directly implies the convergence of the transport problem. The approach we have taken here is a combination of a few, already worked out, methods (originating in the work of Kolmogorov [10], and later Bogolyubov [3]). More precisely, it is a combination of Kolmogorov's transformation of coordinate system (and its refinement due to Tassa [18]) and Bogolyubov's method for singular perturbations. In particular, the latter implies a convergence rate as $\varepsilon \rightarrow 0$.

To the best of our knowledge, this has not been done previously and hence worth noticing. Such a composition of hybrid techniques - combination of singular perturbations, dynamical systems, and homogenization-gives new insights and opens up for the study of convergence rates for similar problems.

We also want to stress that although our result seems to be new, it does not use any new technique, and most probably if the problem was noticed by others, that have worked with the related transport problem, a similar observation would have been made.

\section{Preliminaries and main results}

We first recall the definition of KBM-functions from [15] Definition 4.2.4.

Definition 1 Consider the function $G(t, x)$ continuous in $t$ and $x$ on $[0, \infty) \times \mathbb{R}^{d}$ such that for some constant $L>0$ there holds

$$
\left|G\left(t, x_{1}\right)-G\left(t, x_{2}\right)\right| \leqslant L\left|x_{1}-x_{2}\right|, \text { for all } t \in[0, \infty), x_{1}, x_{2} \in \mathbb{R}^{d} .
$$

If the average

$$
G^{0}(y)=\lim _{\ell \rightarrow \infty} \frac{1}{\ell} \int_{0}^{\ell} G(\tau, y) \mathrm{d} \tau
$$

exists uniformly in $y$ on compact sets $D \subset \mathbb{R}^{d}$ then we call $G$ a KBM-function (KMB stands for Krylov, Bogolyubov and Mitropolski.) 
We next justify the existence of $G^{0}$ and obtain a refined estimate for $\delta$ under the periodicity assumption on $G$ in $t$-variable.

Lemma 1 Consider the function $G:[0, \infty) \times \mathbb{R}^{d} \rightarrow \mathbb{R}$, continuous in $t \in[0, \infty)$ and $x \in \mathbb{R}$ such that for some constant $L>0$ there holds

$$
\left|G\left(t, x_{1}\right)-G\left(t, x_{2}\right)\right| \leqslant L\left|x_{1}-x_{2}\right|, \text { for all } t \in[0, \infty), x_{1}, x_{2} \in \mathbb{R}^{d} .
$$

Suppose $G(t, x)$ is 1-periodic in $t$, then the limit in (10) exists and consequently $G$ is a KBM-function.

Proof For fixed $y$ we have

$$
\begin{aligned}
\int_{0}^{\ell}\left(G(\tau, y)-G^{0}(y)\right) \mathrm{d} \tau & =\sum_{m=1}^{[\ell]} \int_{m-1}^{m}\left(G(\tau, y)-G^{0}(y)\right) \mathrm{d} \tau+\int_{[\ell]}^{\ell}\left(G(\tau, y)-G^{0}(y)\right) \mathrm{d} \tau= \\
& =\int_{[\ell]}^{\ell}\left(G(\tau, y)-G^{0}(y)\right) \mathrm{d} \tau
\end{aligned}
$$

where $[\ell]$ is the integer part of $\ell>0$ and $G^{0}(y)=\int_{0}^{1} G(\tau, y) \mathrm{d} \tau$. Consequently,

$$
\frac{1}{\ell} \int_{0}^{\ell} G(\tau, y) \mathrm{d} \tau=G^{0}(y)+\frac{1}{\ell} \int_{[\ell]}^{\ell}\left(G(\tau, y)-G^{0}(y)\right) \mathrm{d} \tau \rightarrow G^{0}(y) \text { as } \ell \rightarrow \infty .
$$

The second part that $G$ is KBM follows from Lemma 4.6.4 [15].

Note that for periodic $G$ independent of $x$, we have that $G^{0}(x)=\int_{0}^{1} G(\tau, x) \mathrm{d} \tau$ is constant. The convergence rate for almost periodic $G$ depending only on $x$ variable may be weaker as the example in Sect. 6 shows.

We formulate our main results below starting from the one-dimensional problem.

Theorem $1(d=1)$ Let $G_{\star}(t, x), t \in \mathbb{R}, x \in \mathbb{T}$ be positive, periodic in $x$, such that the function $G(t, x):=G_{\star}(x, t)$ (with swapped variables) is KBM-function and

$$
M:=\sup _{x \in \mathbb{R}, t \geqslant 0} \frac{1}{G_{\star}(t, x)}<\infty .
$$

Let $X^{\varepsilon}$ be the solution to the initial value problem

$$
\left\{\begin{array}{l}
\frac{d X^{\varepsilon}}{d t}=\frac{1}{G_{\star}\left(t, \frac{X^{\varepsilon}}{\varepsilon}\right)}, \\
X^{\varepsilon}(0)=p .
\end{array}\right.
$$

Then there is a Lipschitz continuous function $X^{0}(t)$ such that

$$
\left|X^{\varepsilon}(t)-X^{0}(t)\right| \leqslant C(T) \varepsilon, \quad t \in[0, T]
$$

where $T>0$ is the length of the time interval $t \in[0, T], C(T)$ is a positive constant depending only on $T$ and $G_{\star}$. Furthermore, if $G_{\star}(t, \eta)$ does not depend on $t$ and is periodic in $\eta$, then $X^{0}(t)=p_{0}+\beta$ t for some $p_{0}, \beta \in \mathbb{R}$.

In the proof of Theorem 1, we will use a simple version of Bogolyubov's method, tailored for the Cauchy problem $\frac{\mathrm{d} Y^{\varepsilon}}{\mathrm{d} t}=G\left(\frac{t}{\varepsilon}, Y^{\varepsilon}\right), Y^{\varepsilon}(0)=p$, see [3] \$26, [15] Lemma 4.3.1. It is worthwhile to mention that at some point we swap the arguments of the function $G_{\star}$ such that the resulted function $G$ is KBM.

Next we state our main result for the multidimensional problem. 
Theorem $2(d \geqslant 2)$

(a) Let $d \geqslant 2$ be a periodic scalar function $G: \mathbb{R}^{d} \rightarrow \mathbb{R}$ independent of $t, G \in C^{k}\left(\mathbb{R}^{n}\right)$, and there are positive constants $c_{0}$ and $\kappa, k>d+\kappa+1$ such that $a \in \mathbb{R}^{d}$ is diophantine, i.e.,

$$
|\langle a, m\rangle| \geqslant \frac{c_{0}}{|m|^{d+\kappa}}, \quad \forall m \in \mathbb{Z}^{d} \backslash\{0\} .
$$

Finally, suppose that $F=\frac{a}{G}$ satisfies $(\boldsymbol{F . 1})-(\boldsymbol{F} .3)$. If $X^{\varepsilon}$ is the solution to the Cauchy problem $\frac{d z^{\varepsilon}}{d t}=\frac{a}{G\left(\frac{z^{\varepsilon}}{\varepsilon}\right)}, z^{\varepsilon}(0)=p$, then

$$
\left|z^{\varepsilon}(t)-\left(p+\frac{a}{\mathcal{M}(G)} t\right)\right| \leqslant C \varepsilon, \quad t \geqslant 0
$$

where $\mathcal{M}(G)=f_{\mathbb{T}^{d}} G, \mathbb{T}^{d}$ is the d-dimensional torus, and

$$
C=\frac{d|a|}{c_{0} \pi|\mathcal{M}(G)|} \sum_{m \in \mathbb{Z}^{d} \backslash\{0\}}|m|^{d+\kappa}\left|G_{m}\right|<\infty
$$

where $G_{m}$ is the $m$-th Fourier coefficient of $G$.

(b) Let $d=2$ and $F \in C^{k}$ be independent of t, and 1-periodic in $x$-variable. Let further (F.1)-(F.3) hold and $X^{\varepsilon}$ solves the Cauchy problem

$$
\left\{\begin{array}{l}
\frac{d X^{\varepsilon}}{d t}=F\left(\frac{X^{\varepsilon}}{\varepsilon}\right) \\
X^{\varepsilon}(0)=p .
\end{array}\right.
$$

Let $\gamma$ be the rotation number (see Sect. 1.3) and assume that $a=(1, \gamma)$ satisfies (13) with some constants $C>0$ and $\kappa>0$ such that $k>3+\kappa$. Then there is a linear function $X^{0}(t)=p+B t, B \in \mathbb{R}^{2}$ such that

$$
\left|X^{\varepsilon}(t)-X^{0}(t)\right| \leqslant \hat{C} \varepsilon, \quad t \in[0, \infty)
$$

where $\hat{C}$ depends on $\|\rho F\|_{\infty}, \gamma$ and $\|F\|_{C^{k}}$.

We shall use a number of results from dynamical systems. In particular, in the proof of Theorem 2, we shall employ Kolmogorov's theorem on coordinate transformation $y=f(x)$ [10], see Sect. 1.3. It needs to be mentioned that Kolmogorov's proof is not constructive, i.e., he did not write explicit form of such transformation. In [18] Tassa found a simple argument that renders the explicit form of $f$. Such coordinate transformation exists for $d \geqslant 3$ under various assumptions $[1,11]$.

\section{Proof of Theorem 1}

We first observe that if $G$ is a KBM-function then by Definition 1 the following limit

$$
G^{0}(y)=\lim _{\ell \rightarrow \infty} \frac{1}{\ell} \int_{0}^{\ell} G(s, y) \mathrm{d} s
$$

exists uniformly in $y \in D$ for any compact $D \subset \mathbb{R}$. In particular, the Lipschitz continuity of $G$ translates to $G^{0}$. Next let us derive a scaled version of Bogolyubov's estimate in one dimension. 
Lemma 2 Let $G:[0, \infty) \times \mathbb{R} \rightarrow \mathbb{R}$ be a KBM-function periodic in the first variable. Let $h^{\varepsilon}(\xi)$ be the solution of the Cauchy problem $\frac{d h^{\varepsilon}}{d \xi}=G\left(\frac{\xi}{\varepsilon}, h^{\varepsilon}(\xi)\right), h^{\varepsilon}(0)=p$.

Let $G^{0}$ be as in (16) and $h^{0}$ a unique solution of the Cauchy problem

$$
\frac{d h^{0}}{d \xi}=G^{0}\left(h^{0}\right), \quad h^{0}(0)=p
$$

on the finite interval $\left[0, T_{1}\right]$. Then, as $\varepsilon \rightarrow 0$,

$$
\left|h^{\varepsilon}(\xi)-h^{0}(\xi)\right| \leqslant C(T) \varepsilon, \quad 0 \leqslant \xi \leqslant T<T_{1}
$$

for some constant $C(T)>0$ depending only on $T$.

Remark 3 Note that under the conditions of Theorem 1 the solution $h^{0}$ is unique because $G^{0}$ is Lipschitz.

Proof We use Bogolyubov's estimate for the slowly varying systems. Define $\theta^{\varepsilon}(\xi)=h^{\varepsilon}(\varepsilon \xi)$ then we have

$$
\left\{\begin{array}{l}
\frac{\mathrm{d} \theta^{\varepsilon}}{\mathrm{d} \xi}=\varepsilon G\left(\xi, \theta^{\varepsilon}\right) \\
\theta^{\varepsilon}(0)=p
\end{array}\right.
$$

Furthermore, let $\theta^{0}(\xi)$ solve

$$
\left\{\begin{array}{l}
\frac{\mathrm{d} \theta^{0}}{\mathrm{~d} \xi}=\varepsilon G^{0}\left(\theta^{0}\right) \\
\theta^{0}(0)=p
\end{array}\right.
$$

where $G^{0}$ is as in (16). Applying Bogolyubov's estimate, [4] Theorem 12.1 and Remark 12.1, (see also [15] Theorem 4.5.5) to $\theta^{\varepsilon}, \theta^{0}$ we have that

$$
\sup _{\xi \in\left[0, \frac{T}{\varepsilon}\right]}\left|\theta^{\varepsilon}(\xi)-\theta^{0}(\xi)\right| \leqslant C(T) \varepsilon
$$

where $C(T)>0$ depends only on $T$. After setting $h^{0}(\varepsilon \xi)=\theta^{0}(\xi)$, substituting $\varepsilon \xi=s$ in (20) the result follows.

Now we are ready to finish the proof of Theorem 1. Observe that $0<\frac{\mathrm{d} X^{\varepsilon}}{\mathrm{d} t} \leqslant$ $\sup _{x \in \mathbb{R}, t \geqslant 0} \frac{1}{G_{\star}(t, x)}=M<\infty$ and therefore $\left\{X^{\varepsilon}\right\}$ is uniformly Lipschitz continuous on every finite interval $[0, T]$. In fact, we have the estimate $\left|X^{\varepsilon}(t)\right| \leqslant|p|+T M, t \in[0, T]$. Furthermore, $X^{\varepsilon}$ is strictly monotone because $G>0$. Thus $X^{\varepsilon}$ has inverse which we denote by $h^{\varepsilon}$,

$$
\xi=X^{\varepsilon}\left(h^{\varepsilon}(\xi)\right)
$$

Rewriting the system for $h^{\varepsilon}$, we have

$$
\frac{1}{\frac{\mathrm{d} h^{\varepsilon}}{\mathrm{d} \xi}}=\frac{1}{G_{\star}\left(h^{\varepsilon}(\xi), \xi / \varepsilon\right)} \quad \Rightarrow \quad \frac{\mathrm{d} h^{\varepsilon}}{\mathrm{d} \xi}=G_{\star}\left(h^{\varepsilon}(\xi), \xi / \varepsilon\right) .
$$

As for the initial condition, we have $h^{\varepsilon}(p)=0$. 
Denote $G(t, x)=G_{\star}(x, t)$, the function with swapped variables. Note that $G$ satisfies all requirements of Lemma 2 (in particular $G$ is periodic in $t$ ), and hence it follows that $h^{\varepsilon} \rightarrow h^{0}$ locally uniformly on $[0, \infty)$ and the homogenized equation is $\frac{\mathrm{d} h^{0}}{\mathrm{~d} \xi}=G^{0}\left(h^{0}\right)$ where

$$
G^{0}(y)=\lim _{\ell \rightarrow \infty} \frac{1}{\ell} \int_{0}^{\ell} G(\tau, y) \mathrm{d} \tau .
$$

Returning to $X^{\varepsilon}$ and using the refined convergence rate (17) for periodic $G$ in $t$ variable, we note that by $(21)$

$$
\xi=X^{\varepsilon}\left(h^{\varepsilon}(\xi)\right)=X^{\varepsilon}\left(\left[h^{\varepsilon}(\xi)-h^{0}(\xi)\right]+h^{0}(\xi)\right)
$$

implying that $\left|\xi-X^{\varepsilon}\left(h^{0}(\xi)\right)\right|=\left|X^{\varepsilon}\left(\left[h^{\varepsilon}(\xi)-h^{0}(\xi)\right]+h^{0}(\xi)\right)-X^{\varepsilon}\left(h^{0}(\xi)\right)\right| \leqslant M \mid h^{\varepsilon}(\xi)-$ $h^{0}(\xi) \mid \leqslant M C(T) \varepsilon$, where the last inequality follows from (17). Hence, $X^{\varepsilon}$ converges uniformly to $X^{0}(t)$, determined by the implicit equation $\xi=X^{0}\left(h^{0}(\xi)\right)$.

Finally, the last part of Theorem 1 follows from the fact that $G^{0}$ is constant for periodic $G_{\star}$ and therefore $X^{0}(t)$ must be linear function of $t$.

\section{Multidimensional problem: Proof of Theorem 2a}

\subsection{Change of variables for $d \geqslant 2$}

Let $\mathrm{d} \mu=\rho \mathrm{d} x$ be the invariant measure of the system $\frac{\mathrm{d} x_{i}}{\mathrm{~d} t}=F_{i}(x)$ where $F(x)=$ $\left(F_{1}(x), \ldots, F_{d}(x)\right), x \in \mathbb{R}^{d}$ is the vector field on the right hand side of the Eq. (7). If $d=2, F_{1}, F_{2}, \rho \in C^{\infty}, F_{i}: \mathbb{R}^{2} \rightarrow \mathbb{R}$ and $F_{1}^{2}+F_{2}^{2}>0$ then Kolmogorov showed that there is a transformation $x \rightarrow y$ such that in the new system of coordinates the equation transforms into the shear flow $\frac{\mathrm{d} y_{1}}{\mathrm{~d} t}=F, \frac{\mathrm{d} y_{2}}{\mathrm{~d} t}=\gamma F$ where $\gamma$ is the rotation number (see Sect. 1.3), and $F$ is a positive function. Furthermore, if $\gamma$ is diophantine (see the formulation of Theorem 2 for precise condition), then there is another transformation of $\mathbb{R}^{2}, y \rightarrow u$ such that the system takes the form $\frac{\mathrm{d} u_{i}}{\mathrm{~d} t}=a_{i}, i=1,2$ where $a_{i}$ are constants.

For $d \geqslant 3$, Kolmogorov's theorem has been generalized by Kozlov which we state below without proof, see [11].

Proposition 1 Let $d \geqslant 2$ and $G>0, \frac{1}{G} \in C^{k}, k>d+\kappa+1$ is smooth. If $a=\left(a_{1}, \ldots, a_{d}\right)$ is diophantine in the sense of (13), then there exists a change of variables transforming the system

$$
\frac{d w_{j}}{d t}=\frac{a_{j}}{G\left(w_{1}, \ldots, w_{d}\right)}, \quad j=1, \ldots, d
$$

into the constant coefficient system $\frac{d w_{j}}{d t}=a_{j}$.

It is clear that for the shear flow (23) the density of invariant measure is $\rho=G$.

\subsection{Proof of Theorem $2 \mathrm{a}$}

Proof We shall use the coordinate transformation introduced in [11] Theorem 2: if $u(t)$ solves the shear system $\frac{\mathrm{d} u}{\mathrm{~d} t}=\frac{q}{G(u)}$ with diophantine $q$ then the mapping given by the equations

$$
w_{j}=u_{j}+\frac{q_{j}}{\mathcal{M}(G)} f(u), \quad 1 \leqslant j \leqslant d, \quad u=\left(u_{1}, \ldots, u_{d}\right)
$$


transforms the equation into

$$
\frac{\mathrm{d} w_{j}}{\mathrm{~d} t}=\frac{q_{j}}{\mathcal{M}(G)}
$$

as stated in Proposition 1, see [11] page 197. Here $\mathcal{M}(G)=\underset{\mathbb{T}^{d}}{f} G$ is the mean value of $G$ and $f$ is determined from the first-order differential equation

$$
\langle\nabla f, q\rangle=G(u)-\mathcal{M}(G) .
$$

In fact, this mapping is non-degenerate (i.e., has nontrivial Jacobian) and is one-to-one [11]. Taking $\varepsilon u=z$ we see that

$$
\frac{\mathrm{d} u}{\mathrm{~d} t}=\frac{q}{G(u)}
$$

with $q=\frac{a}{\varepsilon}$. From Fourier's expansion we have

$$
G(u)-\mathcal{M}(G)=\sum_{m \in \mathbb{Z}^{d} \backslash\{0\}} G_{m} e^{2 \pi i\langle m, u\rangle}
$$

which by integration gives

$$
f(u)=\sum_{m \in \mathbb{Z}^{d} \backslash\{0\}} \frac{G_{m}}{2 \pi i\langle m, q\rangle} e^{2 \pi i\langle m, u\rangle}=\varepsilon \sum_{m \in \mathbb{Z}^{d} \backslash\{0\}} \frac{G_{m}}{2 \pi i\langle m, a\rangle} e^{2 \pi i\langle m, u\rangle},
$$

and the series is absolutely convergent, due to the assumption that $a$ is diophantine (see (13)) and $G \in C^{k}, k>d+\kappa+1$. In particular, $\left|G_{m}\right| \leqslant C(k, d)(1+|m|)^{-k}$ for some universal constant $C(k, d)>0$ depending only on $d$ and $k$. Notice that the sum is bounded because $\frac{1}{G}$ satisfies the assumptions (F.1)-(F.3). Summarizing we have

$$
\begin{aligned}
w_{j}^{\varepsilon}(t) & =\frac{q_{j}}{\mathcal{M}(G)} t+w_{j}^{\varepsilon}(0) \\
& =\frac{a_{j}}{\varepsilon \mathcal{M}(G)} t+w_{j}^{\varepsilon}(0) .
\end{aligned}
$$

On the other hand, from (24) and (25)

$$
\begin{aligned}
w_{j}^{\varepsilon}(t) & =u_{j}^{\varepsilon}(t)+\frac{q_{j}}{\mathcal{M}(G)} f\left(u^{\varepsilon}\right) \\
& =\frac{z_{j}^{\varepsilon}(t)}{\varepsilon}+\left\{\frac{a_{j}}{\varepsilon} \frac{1}{\mathcal{M}(G)}\right\} \varepsilon \sum_{m \in \mathbb{Z}^{d} \backslash\{0\}} \frac{G_{m}}{2 \pi i\langle m, a\rangle} e^{2 \pi i\left\langle m, u^{\varepsilon}\right\rangle} \\
& =\frac{z_{j}^{\varepsilon}(t)}{\varepsilon}+\frac{a_{j}}{\mathcal{M}(G)} \sum_{m \in \mathbb{Z}^{d} \backslash\{0\}} \frac{G_{m}}{2 \pi i\langle m, a\rangle} e^{\frac{2 \pi i}{\varepsilon}\left\langle m, z^{\varepsilon}\right\rangle} .
\end{aligned}
$$


Combining (26), (27) and $w_{j}^{\varepsilon}(0)=\frac{z_{j}^{\varepsilon}(0)}{\varepsilon}+\frac{q_{j}}{\mathcal{M}(G)} f\left(\frac{z^{\varepsilon}(0)}{\varepsilon}\right)$, which follows from (24), we get $\frac{a_{j}}{\mathcal{M}(G)} t+p_{j}-z_{j}^{\varepsilon}(t)=\sigma(\varepsilon)$ where

$$
\begin{aligned}
\sigma(\varepsilon) & =\varepsilon\left\{-\frac{q_{j}}{\mathcal{M}(G)} f\left(\frac{p}{\varepsilon}\right)+\frac{a_{j}}{\mathcal{M}(G)} \sum_{m \in \mathbb{Z}^{d} \backslash\{0\}} \frac{G_{m}}{2 \pi i\langle m, a\rangle} e^{\frac{2 \pi i}{\varepsilon}\left\langle m, z^{\varepsilon}\right\rangle}\right\} \\
& =\varepsilon\left\{\frac{a_{j}}{\mathcal{M}(G)} \sum_{m \in \mathbb{Z}^{d} \backslash\{0\}} \frac{G_{m}}{2 \pi i\langle m, a\rangle}\left[e^{\frac{2 \pi i}{\varepsilon}\left\langle m, z^{\varepsilon}\right\rangle}-e^{\frac{2 \pi i}{\varepsilon}\langle m, p\rangle}\right]\right\} .
\end{aligned}
$$

Since $G \in C^{k}, k>d+\kappa+1$ and $a$ is diophantine, see (13), it follows that the series $\sum_{m \in \mathbb{Z}^{d} \backslash\{0\}} \frac{\left|G_{m}\right|}{2 \pi|\langle m, a\rangle|}$ converges. Therefore using (13)

$$
|\sigma(\varepsilon)| \leqslant \frac{2 \varepsilon|a|}{|\mathcal{M}(G)|} \sum_{m \in \mathbb{Z}^{d} \backslash\{0\}} \frac{\left|G_{m}\right|}{2 \pi|\langle m, a\rangle|} \leqslant \frac{\varepsilon|a|}{c_{0} \pi|\mathcal{M}(G)|} \sum_{m \in \mathbb{Z}^{d} \backslash\{0\}}|m|^{d+\kappa}\left|G_{m}\right|
$$

and the series converges because from $G \in C^{k}$ we get $\left|G_{m}\right| \leqslant C(k, d)(1+|m|)^{-k}$ with $k>d+\kappa+1$. The proof now follows.

Remark 4 Peirone showed that if $F \in C^{1}\left(\mathbb{T}^{d}\right)$ is $\mathbb{Z}^{d}$ periodic, $u_{0} \in C^{1}$ and the limit $\lim _{t \rightarrow \infty} \frac{S_{F}^{t}(x)}{t}$ exists for a.e. $x \in \mathbb{T}^{d}$ then the problem (2) is homogenizable; see [13] Lemma 2.2 (b). Here $S_{F}^{t}$ is the semigroup generated by (7). Our result establishes the converse of this statement for homogenizable (2).

\section{Proof of Theorem $2 b$}

Our goal here is to apply Kolmogorov's coordinate transformation in order to reduce the general problem to shear flow. For this, Tassa [18] found an explicit formula, that we will write below. We should (again) point out that Kolmogorov's proof in [10] is not constructive.

It is convenient to introduce some basic facts about the equation $\frac{\mathrm{dX}}{\mathrm{d} t}=F(X)$ with $F$ satisfying the properties (F.1)-(F.3). Let $\mathrm{d} \mu=\rho \mathrm{d} x$ be the invariant measure corresponding to this system, then by definition $\operatorname{div}(\rho F)=0$. Thus the vector field $b=\left(b_{1}, b_{2}\right)=$ $\rho F$ is divergence free, 1-periodic, and $\rho \in C^{k}$, see Sect. 1.4. This yields that the integral $\int_{0}^{1} b_{1}\left(x_{1}, x_{2}\right) \mathrm{d} x_{2}$ is constant since

$$
\begin{aligned}
\partial_{x_{1}} \int_{0}^{1} b_{1}\left(x_{1}, x_{2}\right) \mathrm{d} x_{2} & =\int_{0}^{1} \partial_{x_{1}} b_{1}\left(x_{1}, x_{2}\right) \mathrm{d} x_{2} \\
& =-\int_{0}^{1} \partial_{x_{2}} b_{2}\left(x_{1}, x_{2}\right) \mathrm{d} x_{2} \\
& =-\left[b_{2}\left(x_{1}, 1\right)-b_{2}\left(x_{1}, 0\right)\right]=0 .
\end{aligned}
$$

Similarly we have that $\int_{0}^{1} b_{2}\left(x_{1}, x_{2}\right) \mathrm{d} x_{1}$ is constant. Denote $\overline{b_{1}}=\int_{0}^{1} b_{1}\left(x_{1}, x_{2}\right) \mathrm{d} x_{2}, \overline{b_{2}}=$ $\int_{0}^{1} b_{2}\left(x_{1}, x_{2}\right) \mathrm{d} x_{1}$ (which are the mean integrals of $b_{1}, b_{2}$ over $\mathbb{T}^{2}$ ) and set

$$
\begin{aligned}
& y_{1}=f_{1}\left(x_{1}, x_{2}\right)=\frac{1}{\overline{b_{2}}} \int_{0}^{x_{1}} b_{2}(\xi, 0) \mathrm{d} \xi, \\
& y_{2}=f_{2}\left(x_{1}, x_{2}\right)=\frac{1}{\overline{b_{1}}} \int_{0}^{x_{2}} b_{1}\left(x_{1}, \xi\right) \mathrm{d} \xi .
\end{aligned}
$$


It is shown in [18] that in the new coordinate system, we get the shear flow $\frac{\mathrm{d} y}{\mathrm{~d} t}=\frac{a}{G(y)}$ with $a=(1, \gamma)$, where $\gamma$ is the rotation number, see Sect. 1.3. Furthermore, we have that

$$
\left|\frac{\partial\left(y_{1}, y_{2}\right)}{\partial\left(x_{1}, x_{2}\right)}\right|=\frac{b_{1}\left(x_{1}, x_{2}\right)}{\overline{b_{1}}} \frac{b_{2}\left(x_{1}, 0\right)}{\overline{b_{2}}} \neq 0, \quad \forall x \in \mathbb{T}^{2}
$$

and the invariant measure density is

$$
\frac{1}{G(y)}=\frac{b_{2}\left(g_{1}(y), 0\right)}{\overline{b_{2}}} F_{1}\left(g_{1}(y), g_{2}(y)\right)
$$

with $g=\left(g_{1}, g_{2}\right)$ being the inverse of $f=\left(f_{1}, f_{2}\right)$, see [18], page 1395. In particular, it follows

$$
b \in C^{k} \text { and } G \in C^{k}
$$

(recall that $b=\rho F$ and $\rho \in C^{k}$, see Sect. 1.4). Moreover from (29), (30) and the inverse function theorem $g \in C^{k}$ implying $G \in C^{k}, k>3+\kappa$.

In order to take advantage of (29), we introduce the function $z^{\varepsilon}(t)=X^{\varepsilon}(t) / \varepsilon$. Then $z^{\varepsilon}(t)$ solves the Cauchy problem $\frac{\mathrm{d} z^{\varepsilon}}{\mathrm{d} t}=\frac{F\left(z^{\varepsilon}\right)}{\varepsilon}, z^{\varepsilon}(0)=\frac{p}{\varepsilon}$. Clearly, the invariant measure now is $\mathrm{d} \mu_{z}=\frac{1}{\varepsilon} \rho \mathrm{d} z$ and $b^{\varepsilon}=\left(\frac{b_{1}}{\varepsilon}, \frac{b_{2}}{\varepsilon}\right)$ is divergence free. Note that

$$
\frac{b_{i}}{\overline{b_{i}}}=\frac{b_{i}^{\varepsilon}}{\overline{b_{i}^{\varepsilon}}}
$$

and therefore applying the change of variables $y=f(x)$, with mapping $f=\left(f_{1}, f_{2}\right)$ given by (29), we obtain the shear flow

$$
\frac{\mathrm{d} y^{\varepsilon}}{\mathrm{d} t}=a \frac{b_{2}\left(g_{1}\left(y^{\varepsilon}\right), 0\right)}{\overline{b_{2}}} \frac{F_{1}\left(g\left(y^{\varepsilon}\right)\right)}{\varepsilon} .
$$

In order to get rid of $\varepsilon$ in the denominator, we set $w^{\varepsilon}(t)=\varepsilon y^{\varepsilon}(t)$. Then $w^{\varepsilon}(t)$ solves the equation

$$
\frac{\mathrm{d} w^{\varepsilon}}{\mathrm{d} t}=a \frac{b_{2}\left(g_{1}\left(w^{\varepsilon} / \varepsilon\right), 0\right)}{\overline{b_{2}}} F_{1}\left(g\left(w^{\varepsilon} / \varepsilon\right)\right) .
$$

By (29) we have that

$$
\begin{aligned}
& f_{1}\left(x_{1}+1, x_{2}\right)=f_{1}\left(x_{1}, x_{2}\right)+1, \\
& f_{1}\left(x_{1}, x_{2}+1\right)=f_{1}\left(x_{1}, x_{2}\right),
\end{aligned}
$$

and similarly

$$
\begin{aligned}
& f_{2}\left(x_{1}+1, x_{2}\right)=f_{2}\left(x_{1}, x_{2}\right), \\
& f_{2}\left(x_{1}, x_{2}+1\right)=f_{2}\left(x_{1}, x_{2}\right)+1
\end{aligned}
$$

in view of the periodicity of $b$. Consequently if $e_{i},(i=1,2)$ is the unit vector in the canonical basis of $\mathbb{R}^{2}$, then this translates to the inverse of $f$, namely we have $g_{j}(\eta+$ $\left.e_{i}\right)=g_{j}(\eta)+M_{i j}, 1 \leqslant i, j \leqslant 2$ where $M_{i j} \in \mathbb{Z}$, see [18] equation (2.5). This yields that $\frac{1}{G(\eta)}=\frac{b_{2}\left(g_{1}(\eta), 0\right)}{\overline{b_{2}}} F_{1}(g(\eta))$ is periodic function and $w^{\varepsilon}$ solves the Cauchy problem

$$
\frac{\mathrm{d} w^{\varepsilon}}{\mathrm{d} t}=\frac{a}{G\left(\frac{w^{\varepsilon}}{\varepsilon}\right)}, \quad w^{\varepsilon}(0)=\varepsilon f\left(\frac{x^{\varepsilon}(0)}{\varepsilon}\right) .
$$


From here, in light of (29) we have

$$
\begin{aligned}
w_{1}^{\varepsilon}(t) & =\frac{\varepsilon}{\overline{b_{2}}} \int_{0}^{z_{1}^{\varepsilon}(t)} b_{2}(\xi, 0) \mathrm{d} \xi=\frac{\varepsilon}{\overline{b_{2}}} \int_{0}^{x_{1}^{\varepsilon}(t) / \varepsilon} b_{2}(\xi, 0) \mathrm{d} \xi \\
& =x_{1}^{\varepsilon}+\frac{\varepsilon}{\overline{b_{2}}} \int_{0}^{x_{1}^{\varepsilon}(t) / \varepsilon}\left(b_{2}(\xi, 0)-\overline{b_{2}}\right) \mathrm{d} \xi \\
& =x_{1}^{\varepsilon}+\frac{\varepsilon}{\overline{b_{2}}} \int_{\left[x_{1}^{\varepsilon}(t) / \varepsilon\right]}^{x_{1}^{\varepsilon}(t) / \varepsilon}\left(b_{2}(\xi, 0)-\overline{b_{2}}\right) \mathrm{d} \xi
\end{aligned}
$$

for $b$ is periodic; see the proof of Lamma 1 for a similar argument. Here [.] denotes the integer part.

Hence we conclude that

$$
w_{1}^{\varepsilon}(t)=x_{1}^{\varepsilon}(t)+\frac{\varepsilon}{\overline{b_{2}}} \int_{\left[x_{1}^{\varepsilon}(t) / \varepsilon\right]}^{x_{1}^{\varepsilon}(t) / \varepsilon}\left(b_{2}(\xi, 0)-\overline{b_{2}}\right) \mathrm{d} \xi \quad t \in[0, \infty) .
$$

In particular, for the initial condition, we get that $w_{1}^{\varepsilon}(0)=p_{1}+\mathcal{O}(\varepsilon)$. As for the asymptotic expansion of $w_{2}^{\varepsilon}$, we need to use a well-known fact that there is a scalar function $\varphi$ such that $b=\left(\partial_{2} \varphi,-\partial_{1} \varphi\right)$ for every two-dimensional divergence-free vector field $b \in L^{\infty}$. From this equation, it follows $\varphi(x)=\psi(x)+q \cdot x+q_{0}$ where $\psi$ is periodic. Observe that $b=\rho F \in L^{\infty}$ by (F.1)-(F.3) [(in fact $b \in C^{k}$ by (32)], hence $\|\nabla \psi\|_{\infty} \leqslant\|\rho F\|_{\infty}<\infty$. Using this fact, we compute

$$
\begin{aligned}
w_{2}^{\varepsilon}(t)= & \frac{\varepsilon}{\overline{b_{1}}} \int_{0}^{z_{2}^{\varepsilon}(t)} b_{1}\left(\frac{x_{1}^{\varepsilon}}{\varepsilon}, \xi\right) \mathrm{d} \xi=\frac{\varepsilon}{\overline{b_{1}}} \int_{0}^{x_{2}^{\varepsilon}(t) / \varepsilon} b_{1}\left(\frac{x_{1}^{\varepsilon}}{\varepsilon}, \xi\right) \mathrm{d} \xi \\
= & \frac{\varepsilon}{\overline{b_{1}}} \int_{0}^{x_{2}^{\varepsilon}(t) / \varepsilon}\left[b_{1}\left(\frac{x_{1}^{\varepsilon}}{\varepsilon}, \xi\right)-b_{1}(0, \xi)\right] \mathrm{d} \xi+\frac{\varepsilon}{\overline{b_{1}}} \int_{0}^{x_{2}^{\varepsilon}(t) / \varepsilon} b_{1}(0, \xi) \mathrm{d} \xi \\
= & \frac{\varepsilon}{\overline{b_{1}}}\left[\varphi\left(\frac{x_{1}^{\varepsilon}}{\varepsilon}, 0\right)-\varphi\left(\frac{x_{1}^{\varepsilon}}{\varepsilon}, \frac{x_{2}^{\varepsilon}}{\varepsilon}\right)+\varphi\left(0, \frac{x_{2}^{\varepsilon}}{\varepsilon}\right)+\varphi(0,0)\right]+\frac{\varepsilon}{\overline{b_{1}}} \int_{0}^{x_{2}^{\varepsilon}(t) / \varepsilon} b_{1}(0, \xi) \mathrm{d} \xi \\
= & \frac{\varepsilon}{\overline{b_{1}}}\left[\psi\left(\frac{x_{1}^{\varepsilon}}{\varepsilon}, 0\right)-\psi\left(\frac{x_{1}^{\varepsilon}}{\varepsilon}, \frac{x_{2}^{\varepsilon}}{\varepsilon}\right)+\psi\left(0, \frac{x_{2}^{\varepsilon}}{\varepsilon}\right)+\psi(0,0)+2 q_{0}\right] \\
& +x_{2}^{\varepsilon}(t)+\frac{\varepsilon}{\overline{b_{1}}} \int_{\left[x_{2}^{\varepsilon}(t) / \varepsilon\right]}^{x_{2}^{\varepsilon}(t) / \varepsilon}\left(b_{1}(0, \xi)-\overline{b_{1}}\right) \mathrm{d} \xi
\end{aligned}
$$

where the third line follows as in (37), or integrating by parts and using $b=\left(\partial_{2} \varphi,-\partial_{1} \varphi\right)$. In particular, at $t=0$, we have that $w_{2}^{\varepsilon}(0)=p_{2}+\mathcal{O}(\varepsilon)$.

Summarizing, we see that $w^{\varepsilon}$ solves the following Cauchy problem

$$
\frac{\mathrm{d} w^{\varepsilon}}{\mathrm{d} t}=\frac{a}{G\left(\frac{w^{\varepsilon}}{\varepsilon}\right)}, \quad w^{\varepsilon}(0)=\left(p_{1}+\mathcal{O}(\varepsilon), p_{2}+\mathcal{O}(\varepsilon)\right)
$$

where $a=(1, \gamma)$ and $\gamma$ is the rotation number, see Sect. 1.3. By Theorem 2a, for diophantine $a=(1, \gamma)$, there is a linear function $w^{0}$ such that $\left|w^{\varepsilon}(t)-w^{0}(t)\right| \leqslant C \varepsilon, t \in[0, \infty)$. Here $C>0$ depends on $F, \rho$ and $\gamma$ as in Theorem 2a (note that we can apply Theorem 2a because by (32) $G \in C^{k}$ ). Then from (37)

$$
\left|x_{1}^{\varepsilon}(t)-w_{1}^{0}(t)\right| \leqslant \frac{\varepsilon}{\overline{b_{2}}}\left|\int_{\left[x_{1}^{\varepsilon}(t) / \varepsilon\right]}^{x_{1}^{\varepsilon}(t) / \varepsilon}\left(b_{2}(\xi, 0)-\overline{b_{2}}\right) \mathrm{d} \xi\right| \leqslant \frac{2 \varepsilon\|\rho F\|_{L^{\infty}}}{\overline{b_{2}}} .
$$


Finally for $x_{2}^{\varepsilon}$ we have

$$
\begin{aligned}
\left|x_{2}^{\varepsilon}-w_{2}^{0}\right| & \leqslant\left|w_{2}^{\varepsilon}-w_{2}^{0}\right|+\varepsilon\|\psi\|_{L^{\infty}}+\frac{\varepsilon}{\overline{b_{1}}}\left|\int_{\left[x_{2}^{\varepsilon}(t) / \varepsilon\right]}^{x_{2}^{\varepsilon}(t) / \varepsilon}\left(b_{1}(0, \xi)-\overline{b_{1}}\right) \mathrm{d} \xi\right| \\
& =\hat{C} \varepsilon+\varepsilon\|\psi\|_{L^{\infty}}+\varepsilon \frac{2\|\rho F\|_{L^{\infty}}}{\overline{b_{1}}}
\end{aligned}
$$

and the desired estimate follows

\section{Examples}

Example 1 Let $F$ be 1-periodic vector field such that $F_{2}=1$ and

$$
F_{1}\left(x_{1}, x_{2}\right)=F_{1}\left(x_{1}\right)= \begin{cases}1 & 0<x_{1} \leqslant 1 / 2, \\ 0 & 1 / 2<x_{1} \leqslant 1 .\end{cases}
$$

Let $X^{\varepsilon}(t)$ be the solution to the following initial value problem

$$
\left\{\begin{array}{l}
\frac{\mathrm{d} X^{\varepsilon}}{\mathrm{d} t}=F\left(\frac{X^{\varepsilon}}{\varepsilon}\right) \\
X^{\varepsilon}(0)=p
\end{array}\right.
$$

Let $S_{\varepsilon}$ be the (cigar-shaped) $\frac{\varepsilon}{2 \sqrt{2}}$-neighborhood of the ray $p+s E, E=(2,1), s \geqslant 0$, i.e.,

$$
S_{\varepsilon}=\left\{x \in \mathbb{R}^{2}:|x-[p+s E]| \leqslant \frac{\varepsilon}{2 \sqrt{2}}, s \geqslant 0\right\} .
$$

Thus as $\varepsilon \rightarrow 0$ the trajectory (i.e., curves determined by $X^{\varepsilon}$ ) converges to the line $\ell(s)=$ $p+s(2,1), s \geqslant 0$ in Hausdorff distance. Hence the trajectory of the limit is the line $\ell(s)$. As for the speed of the convergence, we note first that by definition $x_{2}^{\varepsilon}=1$, and it is enough to study the ode $\frac{\mathrm{d} z}{\mathrm{~d} t}=F_{1}(z / \varepsilon)$. Multiplying both sides of this equation by $\frac{\mathrm{d} z^{\varepsilon}}{\mathrm{d} t}$ and integrating, we obtain that

$$
\int_{0}^{s}\left|\frac{\mathrm{d} z^{\varepsilon}(t)}{\mathrm{d} t}\right|^{2} \mathrm{~d} t=\int_{0}^{s} F_{1}\left(\frac{z^{\varepsilon}(t)}{\varepsilon}\right) \frac{\mathrm{d} z^{\varepsilon}(t)}{\mathrm{d} t} \mathrm{~d} t=q s+\mathcal{O}(\varepsilon)
$$

where $q=f_{[0,1]} F_{1}=\frac{1}{2}$. Since $\left|\frac{\mathrm{d} z^{\varepsilon}(t)}{\mathrm{d} t}\right| \leqslant \sup F_{1}=1$, we can use a customary compactness argument and infer from Lebesgue's dominated convergence theorem

$$
\int_{0}^{s}\left|\frac{\mathrm{d} z^{0}(t)}{\mathrm{d} t}\right|^{2} \mathrm{~d} t=\frac{s}{2}
$$

where $z^{0}$ is the limit function. After differentiation we get $\left|\frac{\mathrm{d} z^{0}(t)}{\mathrm{d} t}\right|=\sqrt{\frac{1}{2}}$.

The astute reader has probably noticed that we did not use condition (F.3) here, but could still obtain a convergence rate. This is due to the one-dimensional character of the problem, since $F_{2}=1$ here.

Example 2 (One dimension) Another example is given by $F$ with saw-like graph

$$
F(\tau)= \begin{cases}\frac{2 h \tau}{a}+\sigma & \text { if } \tau \in\left[0, \frac{a}{2}\right), \\ \frac{2 h}{a}(a-\tau)+\sigma & \text { if } \tau \in\left[\frac{a}{2}, a\right),\end{cases}
$$




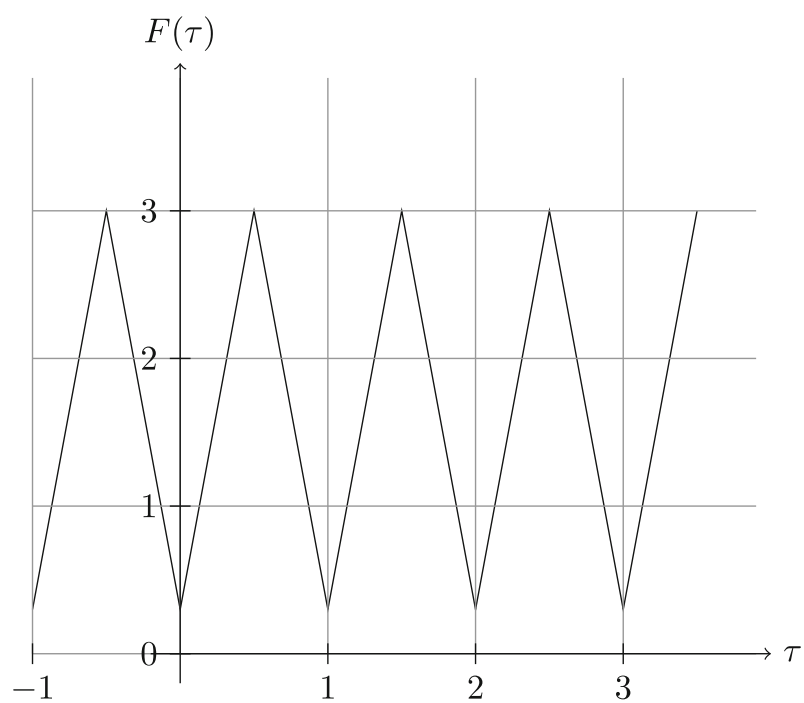

Fig. 1 In this example $a=1, h=3$ and $\sigma=\frac{1}{3}$

periodically extended over $\mathbb{R}$, see Fig. 1 . Here $\sigma>0, a>0$ is the periodicity of $F$ and $h=\max F$ is the peak of $F$. We can solve this equations explicitly: Indeed, we have that

$$
\frac{\mathrm{d} y^{\varepsilon}}{\mathrm{d} t}= \begin{cases}\frac{2 h}{a}\left(\frac{y^{\varepsilon}}{\varepsilon}-k a\right)+\sigma & \text { if } \frac{y^{\varepsilon}}{\varepsilon} \in a k+\left[0, \frac{a}{2}\right), \\ \frac{2 h}{a}\left(a(k+1)-\frac{y^{\varepsilon}}{\varepsilon}\right)+\sigma & \text { if } \frac{y^{\varepsilon}}{\varepsilon} \in a k+\left[\frac{a}{2}, a\right) .\end{cases}
$$

After integration one gets

$$
y^{\varepsilon}= \begin{cases}C_{-}(k) e^{\frac{2 h t}{\varepsilon a}}+\varepsilon k a-\frac{a \sigma \varepsilon}{2 h} & \text { if } y^{\varepsilon} \in \varepsilon a k+\left[0, \frac{a \varepsilon}{2}\right), \\ C_{+}(k) e^{-\frac{2 h t}{\varepsilon a}}+\varepsilon(k+1) a-\frac{a \sigma \varepsilon}{2 h} & \text { if } y^{\varepsilon} \in \varepsilon a k+\left[\frac{a \varepsilon}{2}, a \varepsilon\right),\end{cases}
$$

with some constants $C_{ \pm}(k)$ and $k \in \mathbb{Z}$. Clearly this solution $y^{\varepsilon}$ is monotone and hence the argument using the inverse function in the proof of Lemma 2 works here too. Obviously $\frac{1}{a} \int_{0}^{a} \frac{\mathrm{d} \tau}{F(\tau)}=\frac{a}{h} \log \left(\frac{h+\sigma}{\sigma}\right) \equiv \beta$ and therefore we infer that $y^{\varepsilon}$ converges uniformly to $y^{0}(t)=$ $p+\frac{t}{\beta}$ on any finite closed interval $[0, T]$.

Example 3 Theorem 1 is still valid if the periodicity of $G(\cdot, x)$ is replaced with almost periodicity in $x$ because we needed periodicity in the proof only for the convergence rate for $G^{0}$. In this case one may get weaker error estimates, see [19] Example 11.13. Indeed, the function $F(x)=\sum_{k=0}^{\infty} \frac{1}{(2 k+1)^{2}} \sin \left(\frac{x}{2 k+1}\right)$ is almost periodic. By direct computation

$$
\begin{aligned}
\int_{0}^{T} F(x) \mathrm{d} x & =\sum_{k=0}^{\infty} \frac{2}{2 k+1} \sin ^{2}\left(\frac{T}{2(2 k+1)}\right) \\
& =\sum_{0}^{N(\varepsilon)} \cdots+\sum_{N(\varepsilon)}^{\infty} \cdots
\end{aligned}
$$

$N(\varepsilon) \sim \frac{1}{\varepsilon}$ then in this case $\left|X^{\varepsilon}(t)-X^{0}(t)\right| \leqslant C(T)(\varepsilon|\log \varepsilon|)$ on finite time intervals [0,T]. 
Example 4 (one-dimensional transport equation) One can apply Theorem 1 to the homogenization of some model transport equations such as

$$
\partial_{t} v^{\varepsilon}+H(x / \varepsilon) \partial_{x} v^{\varepsilon}=0, \quad v^{\varepsilon}(0, x)=v_{0}(x) .
$$

Here $H>0$ is $C^{1}$ smooth periodic function. Let $F=(1, H)$ and $\rho$ be the density of invariant measure, i.e., $\operatorname{div}(\rho F)=0$. Therefore, there is a function $M(t, x)$ solving the system

$$
\left\{\begin{array}{l}
\partial_{t} M=-\rho H(y / \varepsilon) \\
\partial_{x} M=\rho .
\end{array}\right.
$$

The level sets $M=$ const are the characteristics of Eq. (39). Noting that $\partial_{t} M=-\rho H \neq 0$ and applying the inverse function theorem to $M(t, x)=$ const we infer that $x=h^{\varepsilon}(t)$, and therefore, for the solution of the Cauchy problem, we have the formula

$$
v^{\varepsilon}(t, x)=v_{0}\left(x-h^{\varepsilon}(t)\right),
$$

where by construction $\frac{\mathrm{d} h^{\varepsilon}}{\mathrm{d} t}=H\left(h^{\varepsilon} / \varepsilon\right), h^{\varepsilon}(0)=x$. Denote $v^{0}(t, x)=v_{0}\left(x-h^{0}(t)\right)$, where $h^{0}=\lim h^{\varepsilon}$. Thus we have from Theorem 1 the estimate

$$
\begin{aligned}
\left|v^{\varepsilon}(t, x)-v^{0}(t, x)\right| & =\left|v_{0}\left(x-h^{\varepsilon}(t)\right)-v_{0}\left(x-h^{0}(t)\right)\right| \\
& \leqslant\left\|\partial_{x} v_{0}\right\|_{\infty}\left|h^{\varepsilon}(t)-h^{0}(t)\right| \leqslant C(T) \varepsilon
\end{aligned}
$$

on finite time intervals $[0, T]$.

Open Access This article is distributed under the terms of the Creative Commons Attribution 4.0 International License (http://creativecommons.org/licenses/by/4.0/), which permits unrestricted use, distribution, and reproduction in any medium, provided you give appropriate credit to the original author(s) and the source, provide a link to the Creative Commons license, and indicate if changes were made.

\section{References}

1. Arnold, V.I.: Polyintegrable flows. Algebra Anal. 4(6), 54-62 (1992)

2. Bardi, M., Cesaroni, A., Scotti, A.: Convergence in multiscale financial models with non-Gaussian stochastic volatility. ESAIM Control Optim. Calc. Var. 22(2), 500-518 (2016)

3. Bogolyubov, N.N., Mitropolski, Y.A.: Asymptotic Methods in the Theory of Non-linear Oscillations, Translated from Russian. Gordon and Breach, New York (1961)

4. Chechkin, G.A., Piatnitski, A.L., Shamaev, A.S.: Homogenization: Methods and Applications (Translations of Mathematical Monographs). AMS, Providence, Rhode Island (2007)

5. Dalibard, A.L.: Homogenization of linear transport equations in a stationary ergodic setting. Commun. Partial Differ. Equ. 33, 881-921 (2008)

6. De Giorgi, E.: On the convergence of solutions of some evolution differential equations. Set Valued Anal. 2(1-2), 175-182 (1994)

7. DiPerna, R.J., Lions, P.-L.: Ordinary differential equations, transport theory and Sobolev spaces. Invent. Math. 98(3), 511-547 (1989)

8. Hou, T., Xin, X.: Homogenization of linear transport equations with oscillatory vector fields. SIAM J. Appl. Math. 52(1), 34-45 (1992)

9. Ibrahim, H., Monneau, R.: On the rate of convergence in periodic homogenization of scalar first-order ordinary differential equation. SIAM J. Math. Anal. 42(5), 2155-2176 (2010)

10. Kolmogorov, A.N.: On dynamical systems with an integral invariant on the torus. Doklady 93(5), 763-766 (1953)

11. Kozlov, V.V.: Dynamical systems with multivalued integrals on a torus. Proc. Steklov Inst. Math. 256(1), 188-205 (2007)

12. Menon, G.: Gradient systems with wiggly energies and related averaging problems. Arch. Ration. Mech. Anal. 162(3), 193246 (2002) 
13. Peirone, R.: Convergence of solutions of linear transport equations. Ergodic Theory Dyn. Syst. 23, 919933 (2003)

14. Piccinini, L.: Homogeneization problems for ordinary differential equations. Rend. Circ. Mat. Palermo (2) 27, 95-112 (1978)

15. Sanders, J., Verhulst, F., Murdock, J.: Averaging Methods in Nonlinear Dynamical Systems. Springer, Berlin (2007)

16. Sinai, Ya.: Introduction to Ergodic Theory. Princeton University Press, Princeton (1976)

17. Tartar, L.: Nonlocal effects induced by homogenization. In: Culumbini, F., et al. (eds.) PDE and Calculus of Variations, pp. 925-938. Birkhäuser, Boston (1989)

18. Tassa, T.: Homogenization of two-dimensional linear flows with integral invariance. SIAM J. Appl. Math. 57(5), 1390-1405 (1997)

19. Verhulst, F.: Methods and Applications of Singular Perturbations, Texts in Applied Mathematics, vol. 50. Springer, Berlin (2010)

20. Weinan, E.: Homogenization of linear and nonlinear transport equations. Commun. Pure Appl. Math. 45(3), 301-326 (1992) 